\title{
Therapeutic approaches for COVID-19 based on the dynamics of interferon- mediated immune responses
}

Farbod Shahabi nezhad ${ }^{\text {a,1,2,3,4 }}$, Pouria Mosaddeghi a,1,2,3,4, Manica Negahdaripour ${ }^{1,2, *}$, Zahra Dehghani $^{1}$, Mitra Farahmandnejad ${ }^{1,2,3,4}$, Mohammad Javad Taghipour ${ }^{1,2,3,4}$, Mohsen Moghadami ${ }^{5}$, Navid Nezafat ${ }^{1,2}$, Seyed Masoom Masoompour ${ }^{5}$

a. Co-first authors in alphabetical order

1 Pharmaceutical Sciences Research Center, Shiraz University of Medical Sciences, Shiraz, Iran

2 Department of Pharmaceutical Biotechnology, School of Pharmacy, Shiraz University of Medical Sciences, Shiraz, Iran

3 Student Research Committee, Shiraz University of Medical Sciences, Shiraz, Iran

4 Cellular and Molecular Medicine Student Research Group, School of Medicine, Shiraz University of Medical Science, Shiraz, Iran

5 Non-Communicable Diseases Research Center, Shiraz University of Medical Sciences, Shiraz, Iran 


\begin{abstract}
As the outbreak of COVID-19 has accelerated, an urgent need for finding strategies to combat the virus is growing. Thus, gaining more knowledge on the pathogenicity mechanism of SARS-CoV2, the causing agent of COVID-19, and its interaction with the immune system is of utmost importance. Although this novel virus is not well known yet, its structural and genetic similarity with SARS-CoV as well as the comparable pattern of age-mortality relations suggest that the previous findings on SARS can be applicable for COVID-19. Therefore, a systems biology study was conducted to investigate the underlying mechanism for the differences in the age-specific mortality of SARS and the most important signaling pathways activated by the virus. The results were then validated through a literature review on COVID-19 and the other closely related viruses, SARS and MERS.

Interferons have shown to possess a crucial role in the defense against coronavirus diseases. The virus can impede the interferon induction in humans. Moreover, STAT1, a key protein in the interferon mediated immune response, is antagonized by the virus. This could explain the increased response threshold of immune cells to IFNs during CoV infections.

A vivid correlation between the innate immune response threshold and the fatality rates in COVID19 can be found. Differences in the dynamics of the interferons-related innate immune responses in children, adults and elderly may explain the reported fatality rates. The increased mortality rates in the elderly can be explained by the higher threshold of interferon-mediated immune responses. Earlier induction of interferons in children and their less developed immune system could be the reason behind their zero or near to zero fatality rate. Administration of interferon-inducing agents, such as Poly (ICLC), could reduce the mortality of SARS at the very early stages of the disease. Adding interferon- $\gamma$ to an interferon-I, as a synergistic combination therapy, might maximize the benefits.

At the later stages of the disease, however, the balance of the immune reactions would be disrupted and the responses would shift toward immnopathogenic over-reactions and probably cytokine storm. Moderating the activity of the immune system and supportive care in such conditions might be the optimum approach.
\end{abstract}

Keywords: 2019 novel coronavirus infection, coronavirus, SARS-CoV, interferon, systems biology 


\section{Introduction}

Coronaviruses (CoVs) are a group of RNA viruses that have the largest RNA genome among all the viruses known to date $(1,2)$. Their genome is surrounded by a bilayer lipid envelope containing the spike and membrane proteins (3). CoVs replicate by the attachment of their spike protein to the host cell receptors resulting in release of the viral genome into the cell (4). They have several hosts including animals and human (5). They mainly cause respiratory disease and common cold (6), but can also cause central nervous system (CNS) infection (7).

The recent outbreak of COVID-19, the new disease caused by a novel coronavirus species, named SARS-CoV-2, has alerted many researchers around the world to find treatment for this condition. Severe acute respiratory syndrome coronavirus (SARS-CoV) and Middle East respiratory syndrome coronavirus (MERS-CoV), two other known viruses from the same genera, were identified in 2002 and 2012, respectively, causing serious respiratory ailments (8).

COVID-19, though a new virus, seems to have a similar pattern to SARS and MERS (9). Despite the differences in the mortality and epidemiological rates of these three diseases, the pattern of age-specific mortality is similar; and the mortality rates get higher as the age increases with in the highest mortality rates among the elderly (9) (Table 1).

Table 1. COVID-19 fatality rate by age, as stated by the "Worldometers" website (10)

\begin{tabular}{|l|l|}
\hline Age & Death rate* \\
\hline 80+ years old & $14.8 \%$ \\
\hline $\mathbf{7 0 - 7 9}$ years old & $8.0 \%$ \\
\hline 60-69 years old & $3.6 \%$ \\
\hline $\mathbf{5 0 - 5 9}$ years old & $1.3 \%$ \\
\hline $\mathbf{4 0 - 4 9}$ years old & $0.4 \%$ \\
\hline $\mathbf{3 0 - 3 9}$ years old & $0.2 \%$ \\
\hline $\mathbf{2 0 - 2 9}$ years old & $0.2 \%$ \\
\hline $\mathbf{1 0 - 1 9}$ years old & $0.2 \%$ \\
\hline $\mathbf{0 - 9}$ years old & no fatalities \\
\hline
\end{tabular}

*Death Rate $=($ number of deaths/number of cases $)=$ probability of dying if infected by the virus $(\%)$. This probability differs depending on the age group. The percentage shown below does NOT represent in any way the share of deaths by age group. Rather, it represents, for a person in a given age group, the risk of dying if infected with COVID-19. 
The zero fatality in children under nine years old (Table 1) seems contradictory to the fact that the immune system gets stronger when a child grows up (11). However, there are differences at the timing of the initiation of immune responses in children versus adults (11).

It has been shown that the potential first lines of defense against SARS are mediated through mannose-binding lectin as a pattern recognition molecule (PRM) of innate immunity (12). Additionally, interleukin (IL)-12 seems to play a vital role in SARS (13). IL-12 activation would lead to the induction of interferons (IFNs) (14). IFN- $\gamma$ is a key moderator in linking the innate immunity to adaptive immune responses (13).

IFNs are a group of cytokines, which communicate between cells against pathogens and have a critical role in the immune system, such as activating natural killer (NK) cells and macrophages, in addition to the flu-like symptoms of various diseases. There are three classes of IFNs: I (such as IFN- $\alpha$ and $-\beta)$, II (IFN- $\gamma$ ), and III, all of which play roles against viral infections (15).

In SARS-CoV and MERS-CoV, the reaction to viral infections by type I IFNs is suppressed. Both CoVs use variant strategies to decrease type I IFN production. This dampening approach is highly associated with the disease severity and increased mortality (16).

On the other hand, in the lethal cases of SARS-CoV or MERS-CoV infection, the increased influx of inflammatory cells is always observed. In a mouse model of SARS- CoV infection, imbalanced type I IFN and inflammatory cells were shown as the main causes of fatal pneumonia (17).

Understanding the pattern of the immune system induction in adults and children in the CoVassociated respiratory syndromes could help to find treatment strategies for these fatal diseases. Considering the lack of available data on COVID-19, SARS can be a helpful model in this regard. Because SARS-CoV-2 has the highest similarity in structure and nucleotide sequence to SARSCoV among other viruses of this family, showing $96 \%$ and $89.6 \%$ sequence identity in the proteins of their envelope and nucleocapsid, respectively (18).

In this study, to figure out the underlying mechanism for the differences in the age-specific mortality of SARS, the most important signaling pathways activated by the virus will be studied using bioinformatics tools and a systems biology approach (19). The obtained results will be validated through a literature review on SARS, MERS, and COVID-19 to indicate how the dynamics of IFN-mediated antiviral response in adults, elderly, and children could determine the severity of the disease and treatment outcomes. 


\section{Methods}

\section{Identification of the deferentially expressed genes using high-throughput RNA-Seq datasets}

At first, "coronavirus" was searched through iLINCS at http://ilincs.org (20). iLINCS is an integrative online platform that brings together different levels of physiological data and integrates them with a bioinformatics analysis engine aiming to analyze and interpret omics data.

The enquiry identified five non-iLINCS datasets about coronavirus infection. GreinGSE52405 dataset was chosen for further analysis. This dataset contains transcriptomics responses in mice infected with either PR8 (highly pathogenic mouse-adapted Influenza A virus) or MA15 (mouseadapted severe acute respiratory syndrome coronavirus).

Geo RNA-seq experiments Interactive Navigator (GREIN) (http://www.ilincs.org/apps/grein/) (21) was applied to analyze the differences in the gene expression level (signature) between the C57BL/6J mice at four days post-infection with MA15 (three experimental groups) and two control groups.

GREIN is a web application with comprehensive analytical toolbox, which provides manipulation and analysis of RNA-seq data. The obtained results were then exported to iLINCS (20) for further analysis.

\section{Gene set enrichment analysis}

Gene set enrichment analysis is a method to interpret deferentially expressed genes (DEGs) in terms of the affected biological pathways and obtain information regarding signature. Gene ontology (GO) enrichment analysis on DEGs was performed by Enrichr $(22,23)$ method at http://amp.pharm.mssm.edu/Enrichr/.

GO knowledgebase (http://geneontology.org/) contains comprehensive information about the function of genes in three main aspects, including biological process (BP), molecular function (MF), and cellular component (CC).

In addition, pathway enrichment analysis for the top 100 DEGs was done by Enrichr as well. NCATS BioPlanet (https://tripod.nih.gov/bioplanet) (24), Kyoto encyclopedia of genes and genomes (KEGG) (https://www.genome.jp/kegg/) (25), and Reactome (https://reactome.org/) (26) pathway databases were used for pathway enrichment analysis to assess the potential association of the signature with pathways. 


\section{Protein-protein interactions network reconstruction}

In order to find the essential proteins and pathways in the gene set, the protein-protein interaction (PPI) network of the signature was extracted from the International Molecular Exchange Consortium (IMEx) protein interactions database (27) through NetworkAnalyst (https://www.networkanalyst.ca) (28).

NetworkAnalyst is a powerful and user-friendly analytics platform, which assists biologists in the interpretation of systems-level data. This tool was implemented to visualize and analyze the PPInetwork of top 100 DEGs. Noteworthy, in order to control the network size, the minimum network tool was selected amongst network tools, which keeps seed proteins and non-seed proteins that are crucial for network connections.

\section{Results and discussion}

\section{Identification of DEGs between control and experimental groups and enrichment analysis}

The currently available wealth of omics data prompt the researchers to develop tools and algorithms to fully exploit the information contained within these data. The list of the DEGs obtained through iLINCS is represented in the supplementary, Tables S1 (top 100 selected genes) and S2 (complete signature). As seen on Fig. (1), the results of the enrichment analysis indicated that the 100-top selected genes are mostly significantly associated with the immune system, IFN signaling, and viral infections.

The findings of our omics analysis showed good congruence with several studies in which the importance of the IFN signaling pathways in CoV infections was reported (29-31), which supports the validity of our selected approach and the notion that $\mathrm{CoV}$ infection can trigger IFN signaling pathway. 
a)

Interferon alpha/beta signaling

Interferon signaling

Immune system

Immune system signaling by interferons, interleukins, prolactin, and growth hormones

Leptin influence on immune response

Interferon-gamma signaling pathway

Innate immune system

Binding of chemokines to chemokine receptors

T cell receptor regulation of apoptosis

Chernokine signaling pathway

b)

Staphylococcus aureus infection

Influenza A

Hepatitis $C$

NOD-like receptor signaling pathway

Measles

Chemokine signaling pathway

Complement and coagulation cascades

Epstein-Barr virus infection

RIG-I-like receptor signaling pathway

Pertussis

c) 


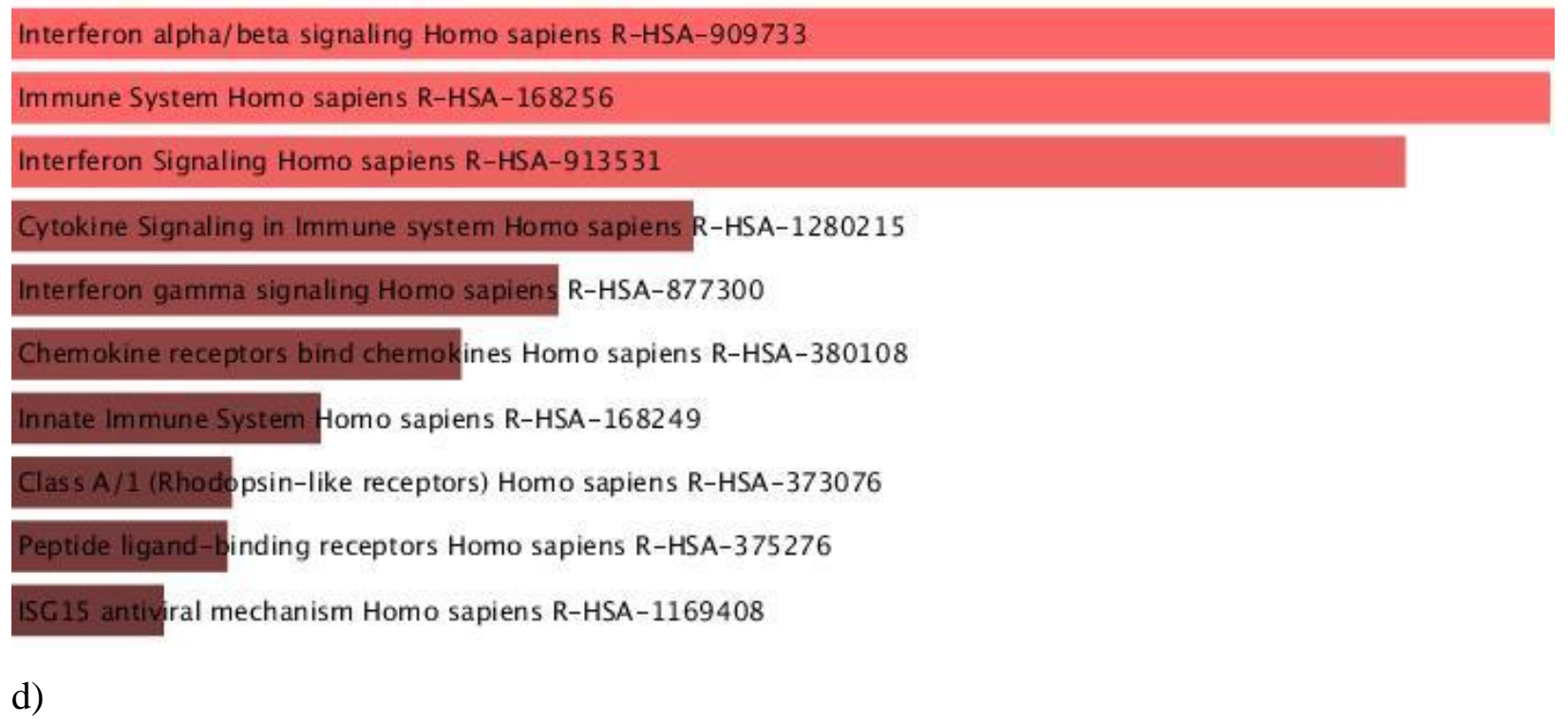

cellular response to type I interferon (GO:0071357)

type I interferon signaling pathway (GO:0060337)

cytokine-mediated signaling pathway (GO:0019221)

cellular response to interferon-gamma (CO:0071346)

positive regulation of leukocyte chemotaxis (GO:0002690)

regulation of viral genome replication (GO:0045069)

cellular response to cytokine stimulus (GO:0071345)

negative regulation of viral genome replication (GO:0045071)

chemokine-mediated signaling pathway (GO:0070098)

positive regulation of monocyte chemotaxis (GO:0090026)

e) 


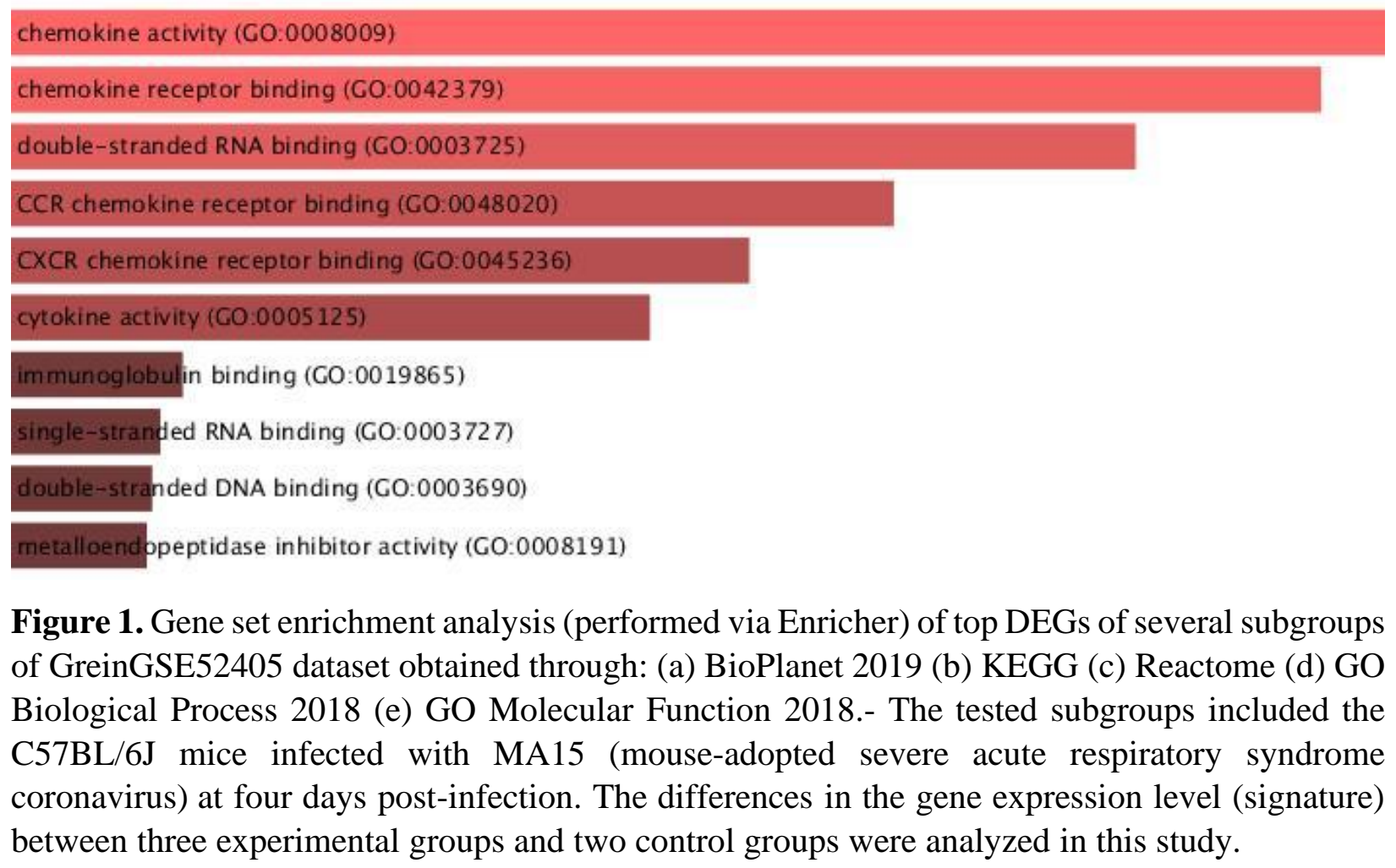

\section{The PPI network of top100 DEGs}

The protein-protein interaction network for the signature was constructed using IMEx protein interactions database through NetworkAnalyst (Fig. 2), representing the crucial proteins in the network, which are called hub proteins. It is well established that the virus-host interaction has a crucial role in the disease outcome, and infecting the host system is mainly mediated through affecting host's important proteins. Though studying the related PPI network can clarify some routes that virus uses in this regard.

The list of all proteins in the network and node centralities (degree and betweenness) is available in Table S3. Noteworthy, the degree of a node is defined as the number of connections that a node has to other nodes and betweenness centrality is the number of the shortest paths passing through the node in a graph (28).

As shown in the PPI network (Fig. 2 and Table S3), STAT1 (Degree: 44, Betweenness: 5916.08), IRF7 (Degree: 18, Betweenness: 1324.53), and ISG15 (Degree:10, Betweenness: 437.65) could be considered as major hub proteins in the network. 
Several studies revealed that $\mathrm{CoV}$ implements some strategies to evade the immune response by antagonizing the arms of IFN signaling pathway (32). In this regard, Frieman et al. indicated that SARS-CoV ORF6 protein blocks the expression of STAT1-activated genes and finally acts as an IFN antagonist (33). Yang and colleagues indicated that MERS-CoV ORF4b antagonizes the antiviral IFN- $\beta$ response by inhibiting IRF3 and IRF7 (34). In addition, it is well established that SARS-CoV and MERS-CoV encode papain-like proteases (PLPs) that are able to impede the immune response function (35). Daczkowski et al. demonstrated that CoV could interact with IFNstimulated gene 15 (ISG15) and antagonize the IFN-mediated antiviral response (36).

Therefore, it seems that coronavirus targets the most important proteins in the IFN signaling pathway to evade the immune system. This highlights the key role of the IFN-mediated antiviral responses in the $\mathrm{CoV}$ infections.

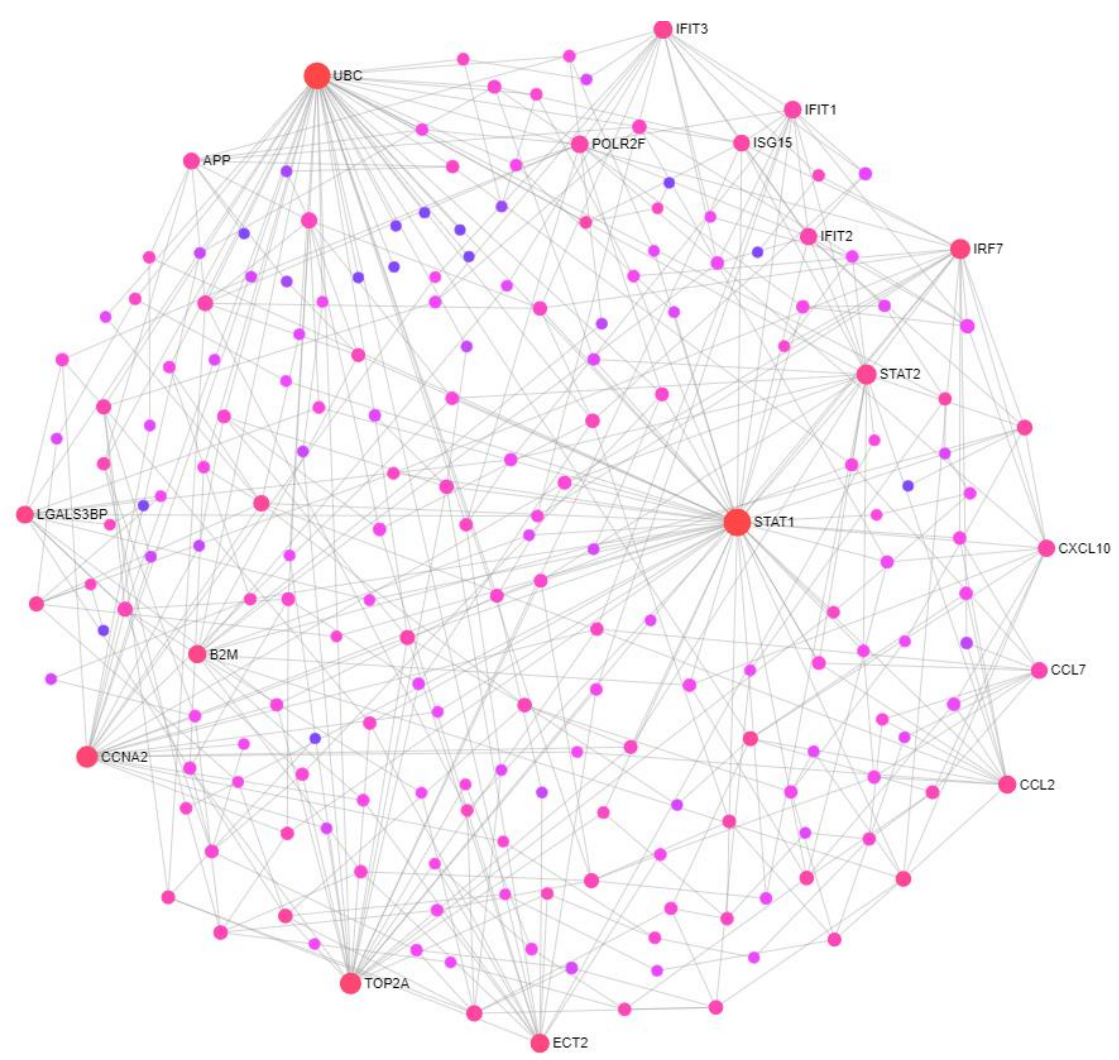

Figure 2. Network analysis of the tested subgroups of GreinGSE52405 dataset (the C57BL/6J mice at four days post-infection with MA15, as three experimental groups, and two control groups). Nodes with bigger size are considered as hubs in this network. 


\section{Dynamics of antiviral response determines the severity of the disease}

The dynamics of IFN-related antiviral responses may be the lost circle in understanding the virulence of CoVs. There are some observations and facts supporting this notion.

The zero fatality in children under nine years old (Table 1) seems contradictory to the fact that the immune system gets stronger when a child grows up.

Considering the immunologic differences between adults and children, the IFN- $\gamma$ induction by NK cells are higher in adults but has a lower threshold in children $(11,37)$. It seems that children respond faster to the virus in the incubation period (38), so that their immune system inhibits the virus replication and prevents high virus titers. On the other hand, in adults, the immunologic response is postponed as the virus impairs the innate immune response by shutting down the signaling pathways.

In a study (39) on the dynamics of the innate immune responses of human cells to SARS-CoV infection, it was indicated that the activation of the IFN regulatory factor (IRF)-3/7 pathway did not occur until 48 hours post-infection. The authors concluded that the delayed IFN-related antiviral response is a possible strategy implemented by $\mathrm{CoV}$ to evade the immune response (40). The virus also circumvents the immune system by hiding its double-stranded RNA in vesicles, causing less IFN induction (32).

In a study by Reghunathan et al. (41), the immunologic responses of 10 adult hospitalized patients with SARS were investigated. It was concluded that the immune response of SARS-affected patients is mainly innate immune response rather than specific antiviral responses. Even though their finding may seem contradictory to the uncovered crucial role of IFN signaling in CoV infections (40) at first, it affirms that SARS-CoV can impede IFN induction and the IFN mediated responses may be suppressed in severe cases of the infection. In other words, the lack of proper antiviral immune responses in the affected patients can be due to antagonization of the sensing and signaling arms of IFN pathways following a high level of virus replication and the consequent impairment in the immune system functions at several days post-infection. Thus, it can be concluded that a delayed IFN-related antiviral response could debilitate the host immunity to inhibit rapid virus replication at the early stages of infection.

This immune system inhibition might happen through affecting STAT1 and other important discussed proteins or some other strategies that $\mathrm{CoV}$ implement to antagonize IFN signaling (31), which finally compromises effective IFN mediated antiviral immune responses. 
To validate this assumption, the dataset of the above-mentioned study (41), GDS1028, was searched and reanalyzed with the help of iLINCS (42). Noteworthy, this dataset contains the expression profiling of peripheral blood mononuclear cells (PBMC) from 10 adult hospitalized patients with SARS and four healthy controls.

The pathway $(24,25,43,44)$ and diseases $(45,46)$ enrichment analysis of the top 100 DEGs was also performed via Enrichr (22,23). Interestingly, disease enrichment analysis demonstrated that the signature was highly associated with some diseases that are the result of the immune system malfunction, including septic shock, obstructive pulmonary bronchitis, allergic diseases, and autoimmune diseases.

Besides, the pathway enrichment analysis revealed that the signature is highly associated with the apoptosis pathway. This result is consistent with the recent study, which represented that the number of T cells in patients with COVID-19 were reduced and functionally exhausted, especially among elderly patients $(\geq 60)$ and in patients requiring Intensive Care Unit (ICU) (47).

The enrichment analysis results are represented in Fig. S2 and Tables S3. Moreover, the list of the DEGs obtained through iLINCS is represented in the supplementary, Tables S4 (top 100 selected genes) and S5 (complete signature).

The importance of $\mathrm{T}$ cell-mediated immune response in respiratory $\mathrm{CoV}$ is well established (48). Type I IFN response is shown crucial in T cell survival $(49,50)$. Moreover, the phosphorylation of STAT1 and STAT5 was increased in the activated naïve CD4+ T cells taken from young adults in order to lower their response threshold to type I IFN stimulation. However, this mechanism was subdued in the elderly naïve CD4+ T cells (51). Likewise, the impaired innate IFN secretion in the elderly is well documented in several studies $(46,48)$.

As mentioned above, SARS-CoV ORF6 protein antagonizes the function of STAT1. Therefore, STAT1 inactivation by ORF6 protein might be the cause of reduced and functionally-exhausted T cells in patients with COVID-19, especially in the elderly patients (33).

Channappanavar et al. (29) have also demonstrated that suboptimal T cell responses occurred in SARS-CoV-infected BALB/c mice. Although the authors concluded that IFN-I-mediated inflammatory responses caused impaired $\mathrm{T}$ cell function, it seems that antagonizing the function of STAT1 by SARS-CoV can play a crucial role in this impairment. Considering the fact that $\mathrm{T}$ cells reduce cytokine storm by modulating the innate immune response (52), it seems that the 
higher response threshold in the elderly, which is aggravated by the antagonizing effects of ORF6 on STAT1 (33), leads to their poor clinical outcome.

Altogether, it might be concluded that the patients with SARS-CoV at the late stages of the disease suffer from many abnormalities, which are the result of immune system imbalance and malfunction and lack of effective IFN-specific immune responses that can lead to proinflammatory reactions and immunopathological conditions, presented by lethal inflammations in the lungs and vascular leakage (29).

\section{Approaches to control COVID19: a systems biology perspective}

Induction of IFNs can play a key role in the body defense against CoVs infections, as supported by several studies mentioned in the following.

Numerous studies have presented the success in defeating CoVs by the direct administration of IFNs. A combination of type I IFN and either IFN- $\gamma$ or IFN- $\lambda$, was shown to synergistically inhibit the virus replication in vitro $(40,53,54)$. Larkin et al. (55) indicated that a combination of IFN- $\alpha$ and IFN- $\gamma$ in vitro provided strong synergistic antiviral activities at much lower dosages of IFN than normally required. Lowering the dose of IFNs in combination therapy offers the advantage of reduction in undesired adverse reactions for the patients. Nagata et al. (56) has described the destructive effect of cytokine storm in adult mice after SARS-CoV infection. While IV injections of TNF- $\alpha$ was not beneficial, intraperitoneal IFN- $\gamma$ injection showed a protective effect. Cinatl $e t$ al. (57) reported the in vitro superiority of IFN- $\beta$ over $-\alpha$ and $-\gamma$, while suggesting the effectiveness of IFN- $\gamma$ over IFN- $\alpha$ in Vero cell cultures of SARS-CoV infection. Bellomi et al. (58) also reported the synergistic effects of IFN- $\gamma$ and $-\beta$ on Vero cells infected with SARS-CoV. Another study, demonstrated that IFN- $\alpha$ and $-\gamma$ co-administration caused hyper-activated IRF-1 and STAT1, which finally led to a more robust antiviral symphony against virus replication (59).

Altogether, it seems that combinational IFN therapy could significantly inhibit virus replication and overcome the increased response threshold of IFN induction that has been resulted by STAT1 inhibition in the immune cells by CoVs, especially in the elderlies

Additionally, another study uncovered that the timing of IFN therapy would be of great importance. In an in vivo study, mice were protected against the virus when IFN-I was given before the maximum rise of the virus, during one day after the infection, though the expression of the ISGs and inflammatory cytokine genes was reduced. On the other hand, treatment failure was seen 
in case of later injection of IFN- $\beta$, because the virus titer went up, and monocytes, macrophages, and neutrophils were accumulated and activated in the lungs, and proinflammatory cytokines were induced, which finally led to severe lethal pneumonia (60).

As another approach, the IFN level can be increased indirectly. Noteworthy, toll like receptor (TLR)3 activation, could induce these IFNs (61-64).

In this regard, two distinct studies $(65,66)$ demonstrated that the pretreatment of mice with TLR3 agonists protected them from mouse-adapted SARS-CoV infection. The used TLR3 agonists, poly ICLC and poly (I:C) (polyinosinic:polycytidylic acid) could augment the production of IFN- $\alpha,-\beta$, and $-\gamma$, which consequently inhibited $\mathrm{CoV}$ replication and compensated for the inhibitory effects of $\mathrm{CoV}$ on IFN signaling pathways (45). Poly (I:C) is a synthetic double-stranded RNA immunostimulant, which is used as adjuvant in vaccine production (67). Poly (I:C) treatment induces not only IFN- $\alpha$ and $-\beta$, but also IFN- $\gamma(65)$. The intranasal or aerosols of poly (I:C) within 48 hours of infection were shown beneficial in mice $(65,66)$. In another study on the infected mice, IFN- $\alpha$ or poly (I:C) were suggested as the only compounds able to inhibit SARS-CoV replication when compared to several different compounds including nelfinavir, calpain inhibitor VI, 3deazaneplanocin A, $\beta$-D-N4-hydroxycytidine, and Alferon ${ }^{\circledR}$ (human leukocyte IFN- $\alpha$-n3) as well as some anti-inflammatory agents, i.e. chloroquine, amodiaquin, and pentoxifylline (68). Interestingly, chloroquine, a recently proposed medication for COVID-19 (69), interacts with poly (I:C) as an endosomal acidification inhibitor, which inhibits poly (I:C)-mediated IFN- $\beta$ expression (70). Therefore, it can be concluded that TLR3 agonists can be a proper option for employment in development of vaccines against COVID-19.

In addition to TLR-3 agonists (poly ICLC (66), poly (I:C) (71), and rintatolimod (32)), some other substances such as TLR7 agonists (e.g. imiquimod) (72), lipopolysaccharides (as TLR4 agonists (73)), and phytohaemagglutinin (PHA) can induce IFN production in the human body as well (74). Nevertheless, some of them may strongly stimulate the immune system and lead to unwanted reactions or toxicity. For example, PHA is a natural compound found in high concentrations in red kidney beans and with lower concentrations in other beans (75). The oral consumption of uncooked red kidney bean has been announced to induce gastrointestinal toxicity and mitogenicity (75) because of high levels of PHA. However, whether a low concentration of PHA could be beneficial at the early stages of the disease or incubation period to stimulate IFN production can be a subject for further research. 
Although IFNs are available as medicinal products, some adverse effects such as bone marrow suppression should be considered for their direct indication (76). Moreover, the protocol for their indication including proper timing and dosing should be confirmed.

All in all, IFN induction in the incubation period and at the very early stages of the disease could be the key to prevent CoV-associated mortalities, yet the proper dosing needs further investigations. Research and clinical trials for finding the right timing for such interventions as well as introducing the proper dose-adjustment protocols are urgently needed.

On the other hand, at the later stages of the disease, the balance of the immune system becomes impaired, hence probable inflammatory over-reactions, cytokine storm, and possible autoimmune responses should be considered. In such circumstances, therapeutic approaches to reduce possible lung inflammations may be needed.

\section{Conclusion}

A vivid correlation between the innate immune response threshold and the fatality rates in COVID19 can be found. The increased mortality rates in the elderly can be explained by the higher threshold of IFN immune responses. Differences in the dynamics of the IFN-related innate immune responses in children, adults, and elderly may explain the different reported fatality rates. Earlier induction of IFNs in children and their less developed immune system could be the reason behind their zero or near to zero mortality.

The key for success in reducing the disease fatality might be the stimulation of the innate immune responses to trigger IFN production at the very early stages of the disease, which might be done through administration of agents that are able to augment IFNs production such as poly ICLC.

Despite the evidences for the efficacy of IFNs in treating CoV-induced infections, the proper dosing and ideal timing for such interventions needs to be verified in clinical trials. Moreover, adding IFN- $\gamma$ to an IFN-I as a combination therapy is strongly suggested.

At the later stages of the disease, the balance of the immune reactions would be disrupted and the responses would shift toward immnopathogenic over-reactions and probably cytokine storm presented by severe respiratory syndrome, which might indicate a need for tempering the immune system activity, although this suggestion might need more clinical evidences. 
All of the stated theories are based on the assumption that the immune response against COVID19 is similar to other coronaviruses, which should be validated through future insights on SARSCoV-2.

\section{Acknowledgment}

This study was supported by Grant from the Research Council of Shiraz University of Medical Sciences, Shiraz University of Medical Sciences, Shiraz, Iran. The authors would like to thank Dr. Mohammadreza Dorvash for his help on selection of the methods.

\section{Conflict of interest}

Authors declare no conflict of interests.

\section{Appendix A. Supplementary data}

Figure S1. Gene set enrichment analysis of top DEGs of GDS1028 dataset.

Table S1. Top 100 selected DEGs (deferentially expressed genes) of some subgroups from GreinGSE52405 dataset.

Table S2. The complete signature of the MA15 group data from GreinGSE52405 dataset.

Table S3. The list of all proteins in the network and node centralities (degree and betweenness) of the tested subgroups of GreinGSE52405 dataset.

Table S4. Top 100 selected DEGs (deferentially expressed genes) of GDS1028 dataset.

Table S5. The complete signature of GDS1028 dataset. 


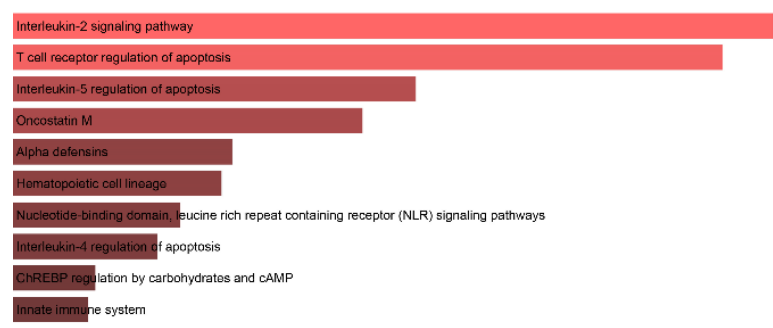

a)

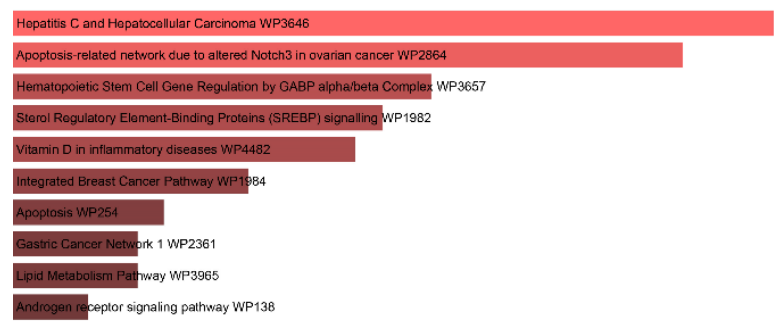

c)

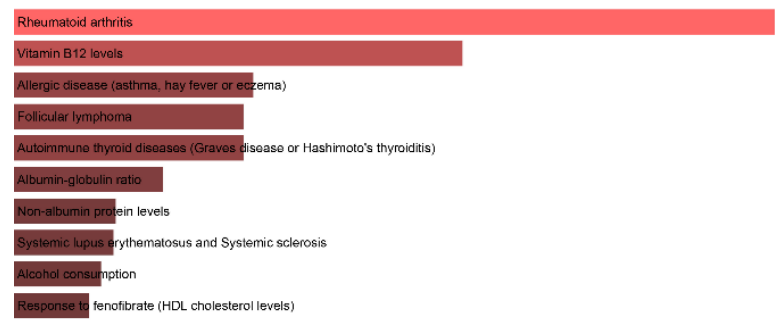

e)

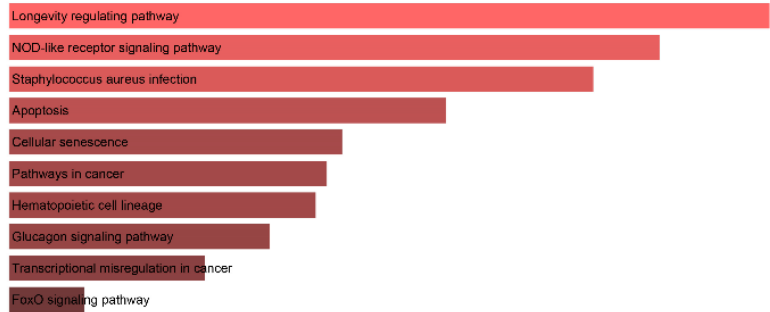

b)

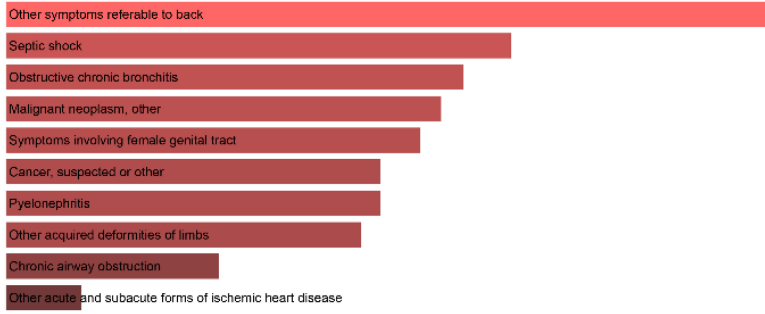

d)

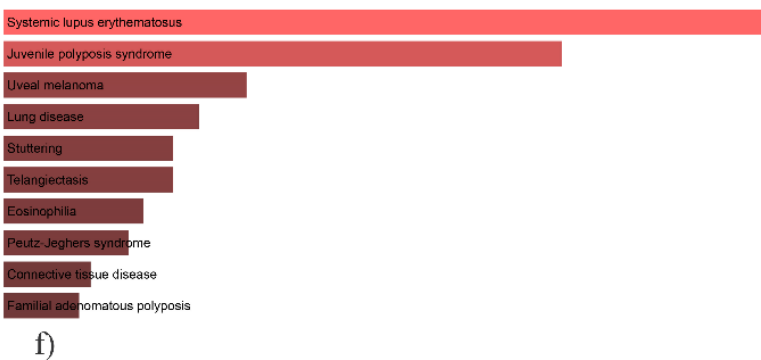

Figure S1. Gene set enrichment analysis of top DEGs of GDS1028 dataset (performed via Enricher). (a) BioPlanet(b) KEGG (c) WikiPathways (d) PheWeb 2019 (e) GWAS catalog 2019 (f) Jensen disease. This dataset contains the expression profiling of peripheral blood mononuclear cells (PBMC) from 10 adult hospitalized patients with SARS and four healthy controls (41).

\section{$\underline{\text { Supplementary tables legends }}$}

Table S1. Top 100 selected DEGs (deferentially expressed genes) of some subgroups from GreinGSE52405 dataset. The data is obtained through iLINCS and analyzed by Grein. The tested subgroups included the C57BL/6J mice infected with MA15 (mouse-adopted severe acute respiratory syndrome coronavirus) at four days post-infection. The differences in the gene expression level (signature) between three experimental groups and two control groups were analyzed in this study.

Table S2. The complete signature of the MA15 group data from GreinGSE52405 dataset obtained through iLINCS and analyzed by Grein. The tested subgroups included the C57BL/6J mice infected with MA15 (mouse-adopted severe acute respiratory syndrome coronavirus) at four days 
post-infection. The differences in the gene expression level (signature) between three experimental groups and two control groups were analyzed in this study.

Table S3. The list of all proteins in the network and node centralities (degree and betweenness) of the tested subgroups of GreinGSE52405 dataset (the C57BL/6J mice at four days post-infection with MA15, as three experimental groups, and two control groups) obtained through analysis of the protein-protein interaction network.

Table S4. Top 100 selected DEGs (deferentially expressed genes) of GDS1028 dataset, obtained through iLINCS. This dataset contains the expression profiling of peripheral blood mononuclear cells (PBMC) from 10 adult hospitalized patients with SARS and four healthy controls (41).

Table S5. The complete signature of GDS1028 dataset, obtained through iLINCS and analyzed by Grein. This dataset contains the expression profiling of peripheral blood mononuclear cells (PBMC) from 10 adult hospitalized patients with SARS and four healthy controls (41). 


\section{References}

1. Ziebuhr J. The coronavirus replicase. Curr Top Microbiol Immunol. 2005;287:57-94.

2. Anand K, Ziebuhr J, Wadhwani P, Mesters JR, Hilgenfeld R. Coronavirus main proteinase (3CLpro) Structure: Basis for design of anti-SARS drugs. Science (80- ). 2003;300(5626):1763-7.

3. de Haan CAM, Smeets M, Vernooij F, Vennema H, Rottier PJM. Mapping of the Coronavirus Membrane Protein Domains Involved in Interaction with the Spike Protein. J Virol. 1999;73(9):7441-52.

4. Weiss SR, Navas-Martin S. Coronavirus Pathogenesis and the Emerging Pathogen Severe Acute Respiratory Syndrome Coronavirus. Microbiol Mol Biol Rev. 2005;69(4):635-64.

5. Lim Y, Ng Y, Tam J, Liu D. Human Coronaviruses: A Review of Virus-Host Interactions. Diseases. 2016;4(4):26.

6. Nicholson KG, Kent J, Ireland DC. Respiratory viruses and exacerbations of asthma in adults. Br Med J. 1993;307(6910):982-6.

7. Bergmann CC, Lane TE, Stohlman SA. Coronavirus infection of the central nervous system: Host-virus stand-off. Vol. 4, Nature Reviews Microbiology. 2006. p. 121-32.

8. Wu Z, McGoogan JM. Characteristics of and Important Lessons From the Coronavirus Disease 2019 (COVID-19) Outbreak in China: Summary of a Report of 72314 Cases From the Chinese Center for Disease Control and Prevention. [Internet]. Jama. 2020 [cited 2020 Mar 2]. Available from: http://www.ncbi.nlm.nih.gov/pubmed/32091533

9. Novel Coronavirus Pneumonia Emergency Response Epidemiology Team. [The epidemiological characteristics of an outbreak of 2019 novel coronavirus diseases (COVID-19) in China]. Zhonghua Liu Xing Bing Xue Za Zhi [Internet]. 2020;41(2):14551. Available from: http://www.ncbi.nlm.nih.gov/pubmed/32064853

10. Coronavirus Age, Sex, Demographics (COVID-19) - Worldometer [Internet]. [cited 2020 Mar 2]. Available from: https://www.worldometers.info/coronavirus/coronavirus-age-sexdemographics/

11. Simon AK, Hollander GA, McMichael A. Evolution of the immune system in humans from infancy to old age. Proc R Soc B Biol Sci. 2015;282(1821):1-8.

12. Ip WKE, Chan KH, Law HKW, Tso GHW, Kong EKP, Wong WHS, et al. MannoseBinding Lectin in Severe Acute Respiratory Syndrome Coronavirus Infection. J Infect 
Dis. 2005;191(10):1697-704.

13. Tang F, Liu W, Zhang F, Xin ZT, Wei MT, Zhang PH, et al. IL-12 RB1 genetic variants contribute to human susceptibility to severe acute respiratory syndrome infection among Chinese. PLoS One. 2008;3(5):e2183.

14. Brown J, Wang H, Hajishengallis GN, Martin M. TLR-signaling networks: An integration of adaptor molecules, kinases, and cross-talk. J Dent Res. 2011;90(4):417-27.

15. Samuel CE. Antiviral actions of interferons. Clin Microbiol Rev. 2001;14(4):778-809.

16. Perlman S, Dandekar AA. Immunopathogenesis of coronavirus infections: Implications for SARS. Vol. 5, Nature Reviews Immunology. 2005. p. 917-27.

17. Channappanavar R, Perlman S. Pathogenic human coronavirus infections: causes and consequences of cytokine storm and immunopathology. Vol. 39, Seminars in Immunopathology. 2017. p. 529-39.

18. Zhou Y, Hou Y, Shen J, Huang Y, Martin W, Cheng F. Network-based Drug Repurposing for Human Coronavirus. medRxiv [Internet]. 2020 Jan 1;2020.02.03.20020263. Available from:

https://www.medrxiv.org/content/medrxiv/early/2020/02/05/2020.02.03.20020263.full.pdf

19. Dorvash M, Farahmandnia M, Mosaddeghi P, Farahmandnejad M, Saber H, Khorraminejad-Shirazi M, et al. Dynamic modeling of signal transduction by mTOR complexes in cancer. J Theor Biol [Internet]. 2019 Dec 21 [cited 2020 Mar 4];483:109992. Available from: http://www.ncbi.nlm.nih.gov/pubmed/31493485

20. Pilarczyk M, Najafabadi MF, Kouril M, Vasiliauskas J, Niu W, Shamsaei B, et al. Connecting omics signatures of diseases, drugs, and mechanisms of actions with iLINCS. bioRxiv [Internet]. 2019;826271. Available from: http://biorxiv.org/content/early/2019/10/31/826271.abstract

21. Mahi N Al, Najafabadi MF, Pilarczyk M, Kouril M, Medvedovic M. GREIN: An Interactive Web Platform for Re-analyzing GEO RNA-seq Data. Sci Rep. 2019;9(1):7580.

22. Kuleshov M V., Jones MR, Rouillard AD, Fernandez NF, Duan Q, Wang Z, et al. Enrichr: a comprehensive gene set enrichment analysis web server 2016 update. Nucleic Acids Res. 2016;44(W1):W90-7.

23. Chen EY, Tan CM, Kou Y, Duan Q, Wang Z, Meirelles G V., et al. Enrichr: Interactive and collaborative HTML5 gene list enrichment analysis tool. BMC Bioinformatics. 
$2013 ; 14$.

24. Huang R, Grishagin I, Wang Y, Zhao T, Greene J, Obenauer JC, et al. The NCATS BioPlanet - An integrated platform for exploring the universe of cellular signaling pathways for toxicology, systems biology, and chemical genomics. Front Pharmacol. 2019;10(APR):445.

25. Ogata H, Goto S, Sato K, Fujibuchi W, Bono H, Kanehisa M. KEGG: Kyoto encyclopedia of genes and genomes. Vol. 27, Nucleic Acids Research. 1999. p. 29-34.

26. Fabregat A, Jupe S, Matthews L, Sidiropoulos K, Gillespie M, Garapati P, et al. The Reactome Pathway Knowledgebase. Nucleic Acids Res. 2018;46(D1):D649-55.

27. Orchard S, Kerrien S, Abbani S, Aranda B, Bhate J, Bidwell S, et al. Protein interaction data curation: The International Molecular Exchange (IMEx) consortium. Vol. 9, Nature Methods. NIH Public Access; 2012. p. 345-50.

28. Zhou G, Soufan O, Ewald J, Hancock REW, Basu N, Xia J. NetworkAnalyst 3.0: a visual analytics platform for comprehensive gene expression profiling and meta-analysis. Nucleic Acids Res. 2019;47(W1):W234-41.

29. Channappanavar R, Fehr AR, Vijay R, Mack M, Zhao J, Meyerholz DK, et al. Dysregulated Type I Interferon and Inflammatory Monocyte-Macrophage Responses Cause Lethal Pneumonia in SARS-CoV-Infected Mice. Cell Host Microbe. 2016;19(2):181-93.

30. Clementz MA, Chen Z, Banach BS, Wang Y, Sun L, Ratia K, et al. Deubiquitinating and Interferon Antagonism Activities of Coronavirus Papain-Like Proteases. J Virol. 2010;84(9):4619-29.

31. Kindler E, Thiel V, Weber F. Interaction of SARS and MERS Coronaviruses with the Antiviral Interferon Response. In: Advances in Virus Research. 2016. p. 219-43.

32. Strayer D, Dickey R, Carter W. Sensitivity of SARS/MERS CoV to Interferons and Other Drugs Based on Achievable Serum Concentrations in Humans. Infect Disord - Drug Targets [Internet]. 2014 Sep 11 [cited 2020 Mar 3];14(1):37-43. Available from: http://www.ncbi.nlm.nih.gov/pubmed/25019238

33. Frieman M, Yount B, Heise M, Kopecky-Bromberg SA, Palese P, Baric RS. Severe Acute Respiratory Syndrome Coronavirus ORF6 Antagonizes STAT1 Function by Sequestering Nuclear Import Factors on the Rough Endoplasmic Reticulum/Golgi Membrane. J Virol. 
2007;81(18):9812-24.

34. Yang Y, Ye F, Zhu N, Wang W, Deng Y, Zhao Z, et al. Middle East respiratory syndrome coronavirus ORF4b protein inhibits type i interferon production through both cytoplasmic and nuclear targets. Sci Rep. 2015;5:17554.

35. Sun L, Xing Y, Chen X, Zheng Y, Yang Y, Nichols DB, et al. Coronavirus papain-like proteases negatively regulate antiviral innate immune response through disruption of STING-mediated signaling. PLoS One. 2012;7(2):e30802.

36. Daczkowski CM, Dzimianski J V., Clasman JR, Goodwin O, Mesecar AD, Pegan SD. Structural Insights into the Interaction of Coronavirus Papain-Like Proteases and Interferon-Stimulated Gene Product 15 from Different Species. J Mol Biol. 2017;429(11):1661-83.

37. Dobbs K, Tabellini G, Calzoni E, Patrizi O, Martinez P, Giliani SC, et al. Natural killer cells from patients with recombinase-activating gene and non-homologous end joining gene defects comprise a higher frequency of CD56bright NKG2A+++ cells, and yet display increased degranulation and higher perforin content. Front Immunol. 2017;8(JUL):798.

38. Ivarsson MA, Loh L, Marquardt N, Kekäläinen E, Berglin L, Björkström NK, et al. Differentiation and functional regulation of human fetal NK cells. J Clin Invest. 2013;123(9):3889-901.

39. Okabe Y, Kawane K, Nagata S. IFN regulatory factor (IRF) 3/7-dependent and independent gene induction by mammalian DNA that escapes degradation. Eur J Immunol [Internet]. 2008 Nov [cited 2020 Mar 4];38(11):3150-8. Available from: http://www.ncbi.nlm.nih.gov/pubmed/18991290

40. Yoshikawa T, Hill TE, Yoshikawa N, Popov VL, Galindo CL, Garner HR, et al. Dynamic innate immune responses of human bronchial epithelial cells to severe acute respiratory syndrome-associated coronavirus infection. PLoS One. 2010;5(1):e8729.

41. Reghunathan R, Jayapal M, Hsu LY, Chng HH, Tai D, Leung BP, et al. Expression profile of immune response genes in patients with severe acute respiratory syndrome. BMC Immunol. 2005;6:2.

42. Pilarczyk M, Najafabadi MF, Kouril M, Vasiliauskas J, Niu W, Shamsaei B, et al. Connecting omics signatures of diseases, drugs, and mechanisms of actions with iLINCS. 
bioRxiv [Internet]. 2019 Oct 31 [cited 2020 Mar 3];826271. Available from: http://biorxiv.org/content/early/2019/10/31/826271.abstract

43. Kanehisa M, Sato Y, Kawashima M, Furumichi M, Tanabe M. KEGG as a reference resource for gene and protein annotation. Nucleic Acids Res. 2016;44(D1):D457-62.

44. Slenter DN, Kutmon M, Hanspers K, Riutta A, Windsor J, Nunes N, et al. WikiPathways: A multifaceted pathway database bridging metabolomics to other omics research. Nucleic Acids Res. 2018;46(D1):D661-7.

45. Buniello A, Macarthur JAL, Cerezo M, Harris LW, Hayhurst J, Malangone C, et al. The NHGRI-EBI GWAS Catalog of published genome-wide association studies, targeted arrays and summary statistics 2019. Nucleic Acids Res. 2019;47(D1):D1005-12.

46. Pletscher-Frankild S, Pallejà A, Tsafou K, Binder JX, Jensen LJ. DISEASES: Text mining and data integration of disease-gene associations. Methods. 2015;74(3):83-9.

47. Diao B, Wang C, Tan Y, Chen X, Liu Y, Ning L, et al. Reduction and Functional Exhaustion of T Cells in Patients with Coronavirus Disease 2019 (COVID-19). medRxiv. 2020 Feb 20;2020.02.18.20024364.

48. Channappanavar R, Zhao J, Perlman S. T cell-mediated immune response to respiratory coronaviruses. Immunol Res. 2014;59(1-3):118-28.

49. Lombardi G, Dunne PJ, Scheel-Toellner D, Sanyal T, Pilling D, Taams LS, et al. Type 1 IFN Maintains the Survival of Anergic CD4 + T Cells . J Immunol. 2000;165(7):3782-9.

50. Marrack P, Kappler J, Mitchell T. Type I interferons keep activated T cells alive. J Exp Med. 1999;189(3):521-9.

51. Li G, Ju J, Weyand CM, Goronzy JJ. Age-Associated Failure To Adjust Type I IFN Receptor Signaling Thresholds after T Cell Activation. J Immunol. 2015;195(3):865-74.

52. Dong Kim K, Zhao J, Auh S, Yang X, Du P, Tang H, et al. Adaptive immune cells temper initial innate responses. Nat Med. 2007;13(10):1248-52.

53. Sainz B, Mossel EC, Peters CJ, Garry RF. Interferon-beta and interferon-gamma synergistically inhibit the replication of severe acute respiratory syndrome-associated coronavirus (SARS-CoV). Virology [Internet]. 2004 Nov 10 [cited 2020 Mar 1];329(1):11-7. Available from: http://www.ncbi.nlm.nih.gov/pubmed/15476870

54. Mossel EC, Sainz B, Garry RF, Peters CJ. Synergistic inhibition of SARS-coronavirus replication by type I and type II IFN. Adv Exp Med Biol. 2006;581:503-6. 
55. Larkin J, Jin L, Farmen M, Venable D, Huang Y, Tan SL, et al. Synergistic antiviral activity of human interferon combinations in the hepatitis $\mathrm{C}$ virus replicon system. $\mathrm{J}$ Interf Cytokine Res [Internet]. 2003 May 1 [cited 2020 Mar 4];23(5):247-57. Available from: http://www.ncbi.nlm.nih.gov/pubmed/12804067

56. Nagata N, Iwata N, Hasegawa H, Fukushi S, Harashima A, Sato Y, et al. Mouse-passaged severe acute respiratory syndrome-associated coronavirus leads to lethal pulmonary edema and diffuse alveolar damage in adult but not young mice. Am J Pathol. 2008 Jun $1 ; 172(6): 1625-37$.

57. Cinatl J, Morgenstern B, Bauer G, Chandra P, Rabenau H, Doerr HW. Treatment of SARS with human interferons. Lancet [Internet]. 2003 Jul 26 [cited 2020 Mar 4];362(9380):2934. Available from: http://www.ncbi.nlm.nih.gov/pubmed/12892961

58. Scagnolari C, Vicenzi E, Bellomi F, Stillitano MG, Pinna D, Poli G, et al. Increased sensitivity of SARS-coronavirus to a combination of human type I and type II interferons. Antivir Ther. 2004;9(6):1003-11.

59. Zhang XN, Liu JX, Hu YW, Chen H, Yuan ZH. Hyper-activated IRF-1 and STAT1 contribute to enhanced interferon stimulated gene (ISG) expression by interferon alpha and gamma co-treatment in human hepatoma cells. Biochim Biophys Acta. 2006;1759(89):417-25.

60. Channappanavar R, Fehr AR, Zheng J, Wohlford-Lenane C, Abrahante JE, Mack M, et al. IFN-I response timing relative to virus replication determines MERS coronavirus infection outcomes. J Clin Invest. 2019;129(9):3625-39.

61. Li J, Ye L, Wang X, Hu S, Ho W. Induction of interferon- $\lambda$ contributes to toll-like receptor 3-mediated herpes simplex virus type 1 inhibition in astrocytes. J Neurosci Res. 2012;90(2):399-406.

62. Matsumoto M, Seya T. TLR3: Interferon induction by double-stranded RNA including poly(I:C). Vol. 60, Advanced Drug Delivery Reviews. 2008. p. 805-12.

63. Negishi H, Osawa T, Ogami K, Ouyang X, Sakaguchi S, Koshiba R, et al. A critical link between Toll-like receptor 3 and type II interferon signaling pathways in antiviral innate immunity. Proc Natl Acad Sci U S A. 2008;105(51):20446-51.

64. Uematsu S, Akira S. Toll-like receptors and type I Interferons. Vol. 282, Journal of Biological Chemistry. 2007. p. 15319-24. 
65. Zhao J, Wohlford-Lenane C, Zhao J, Fleming E, Lane TE, McCray PB, et al. Intranasal Treatment with Poly(I\{middle dot $\}$ C) Protects Aged Mice from Lethal Respiratory Virus Infections. J Virol. 2012;86(21):11416-24.

66. Kumaki Y, Salazar AM, Wandersee MK, Barnard DL. Prophylactic and therapeutic intranasal administration with an immunomodulator, Hiltonol@ (Poly IC:LC), in a lethal SARS-CoV-infected BALB/c mouse model. Antiviral Res. 2017;139:1-12.

67. Ngoi SM, Tovey MG, Vella AT. Targeting Poly(I:C) to the TLR3-Independent Pathway Boosts Effector CD8 T Cell Differentiation through IFN- $\alpha / \beta$. J Immunol. 2008;181(11):7670-80.

68. Barnard DL, Day CW, Bailey K, Heiner M, Montgomery R, Lauridsen L, et al. Evaluation of immunomodulators, interferons and known in vitro SARS-CoV inhibitors for inhibition of SARS-CoV replication in BALB/c mice. Antivir Chem Chemother. 2006;17(5):27584.

69. Gao J, Tian Z, Yang X. Breakthrough: Chloroquine phosphate has shown apparent efficacy in treatment of COVID-19 associated pneumonia in clinical studies. [Internet]. Bioscience trends. 2020. Available from: http://www.ncbi.nlm.nih.gov/pubmed/32074550

70. Kumar A, Zhang J, Yu FSX. Toll-like receptor 3 agonist poly(I:C)-induced antiviral response in human corneal epithelial cells. Immunology. 2006 Jan;117(1):11-21.

71. Perrot I, Deauvieau F, Massacrier C, Hughes N, Garrone P, Durand I, et al. TLR3 and Rig-Like Receptor on Myeloid Dendritic Cells and Rig-Like Receptor on Human NK Cells Are Both Mandatory for Production of IFN- $\gamma$ in Response to Double-Stranded RNA. J Immunol. 2010;185(4):2080-8.

72. Nerurkar L, McColl A, Graham G, Cavanagh J. The Systemic Response to Topical Aldara Treatment is Mediated Through Direct TLR7 Stimulation as Imiquimod Enters the Circulation. Sci Rep. 2017;7(1):16570.

73. Pulendran B, Kumar P, Cutler CW, Mohamadzadeh M, Van Dyke T, Banchereau J. Lipopolysaccharides from Distinct Pathogens Induce Different Classes of Immune Responses In Vivo. J Immunol. 2001;167(9):5067-76.

74. Miyawaki T, Seki H, Taga K, Sato H, Taniguchi N. Dissociated production of interleukin2 and immune $(\gamma)$ interferon by phytohaemagglutinin stimulated lymphocytes in healthy infants. Clin Exp Immunol. 1985;59(2):505-11. 
75. Zhang J, Shi J, Ilic S, Jun Xue S, Kakuda Y. Biological properties and characterization of lectin from red kidney bean (Phaseolus Vulgaris). Food Rev Int. 2009;25(1):12-27.

76. Zoumbos NC, Gascon P, Djeu JY, Young NS. Interferon is a mediator of hematopoietic suppression in aplastic anemia in vitro and possibly in vivo. Proc Natl Acad Sci U S A. 1985;82(1):188-92. 